\title{
Deacylative alkylation (DaA) of $N$-methyl-3-acetyl-2-oxindole for the synthesis of symmetrically 3,3-disubstituted 2-oxindoles. An access gate to anticancer agents and natural products.
}

\author{
CRISTINA MORENO-CABRERIZO ${ }^{1,2}$, AITOR ORTEGA-MARTÍNEZ ${ }^{1,2}$, CYNTHIA \\ MOLINA ${ }^{1,2}$, CARMEN NÁJERA ${ }^{1}$ and JOSÉ M. SANSANO ${ }^{1,2}$ \\ ${ }^{1}$ Departamento de Química Orgánica, Facultad de Ciencias, Universidad de Alicante, \\ Ctra. Alicante S. Vicente, s/n, Apdo. 99, 03080 Alicante, Spain \\ ${ }^{2}$ Instituto de Síntesis Orgánica/ISO, Universidad de Alicante, Ctra. Alicante S. Vicente, s/n, Apdo. 99, 03080 Alicante, Spain
}

Manuscript received on October 9, 2017; accepted for publication on November 16, 2017

\begin{abstract}
The synthesis of 3,3-disubstituted $N$-methyloxindoles, starting from 3-acetyl-2-hydroxy-1-methyloxindole employing a sequential one-pot synthesis, is studied. The process involves a first alkylation in the presence of 1 equiv. of both organic halide and Triton B and the second one employs another 1.5 equiv. of each in moderate to high yields. This procedure is compared with the results obtained from the direct dialkylation of $\mathrm{N}$-methyloxindole. The metathesis of one of the corresponding diallylated product was also studied obtaining the spiranic oxindole. All these methodologies are directed towards the access to anticancer agents and natural biologically active products.
\end{abstract}

Key words: deacylation, alkylation, 2-oxindoles, metathesis, anticancer, natural products.

\section{INTRODUCTION}

Sophisticated (Zhanget al. 2017, Nadege et al. 2016) or simple (Trost and Zhang 2006) natural compounds incorporating a 3,3-disubstituted 2-oxindole are frequently found (Kaur et al. 2016, Saraswat et al. 2016, Fonseca and Cook 2016, Ziarani et al. 2013). In fact, the generation of this quaternary carbon as a consequence of this 3,3-disubstitution is a key point, based in the Ingold-Thorpe effect, for the

Correspondence to: José Miguel Sansano

E-mail: jmsansano@ua.es

Carmen Nájera

E-mail: cnajera@ua.es

* Contribution to the centenary of the Brazilian Academy of Sciences. construction of very complex natural skeletons or drugs (Cao et al. 2014, Shen et al. 2012, Singh and Desta 2012, Dalpozzo et al. 2012, Zhou et al. 2010, Galliford et al.2007). For example, 3,3-disubstituted 2-oxindoles are important class of heterocyclic compounds occurring in natural alkaloids such as the alkaloid horsfiline (Trost et al. 2006), esermethole (Trost et al. 2006), acetylcholinesterase inhibitors physostigmine and phenserine (Huang et al. 2004), and muscle relaxant agents flustramides $\mathrm{A}$ and $\mathrm{B}$ (Trost et al. 2011) (Figure 1). Besides synthetic spiroxindoles, with a rigid heterocyclic ring fused at the 3-position of the oxindole core (Figure 1), become the most effective compounds as inhibitors of the tumor cells proliferation inducing apoptosis 
<smiles>COc1ccc2c(c1)C1(CCN(C)C1)C(=O)N2</smiles><smiles>C=CC(C)(C)C12CC(=O)N(C)C1N(CC=C(C)C)c1cc(Br)ccc12</smiles>

rac-flustramide A<smiles>[X]c1ccc2c(c1)C1(C)CCN(C)C1N2[Y19]</smiles>

esermethole: $X=O M e$ physostigmine: $X=$ OCONHMe phenserine: $X=O C O N H P h$<smiles>[R]N1C(=O)C(C)(CC=C)c2cc(OC)ccc21</smiles><smiles>C=CC1(CC=C(C)C)C(=O)Nc2cc(Br)ccc21</smiles>

rac-flustramide $\mathrm{B}$<smiles>[R]CC1CC([R])C([R])C12C(=O)N([R])c1cc([R])ccc12</smiles>

Figure 1 - Natural and synthetic biologically active compounds containing the 2-oxindole unit.

on them without affecting activities of normal cells (Gupta et al. 2017, Dondas et al. 2017, Saraswat et al. 2016, Yu et al. 2015, David et al. 2016).

Although an unsymmetrical 3,3-substitution is more attractive from the synthetic point of view (Ashimori et al. 1993, Matsuura et al. 1998, Shaughnessy et al. 1998, Kundig et al. 2007, Marsden et al. 2008, Altman et al. 2008, Ruck et al. 2008, Jia and Kundig 2009, Perry and Taylor 2009, Ghosh et al. 2012, Tian et al. 2008, He et al. 2009, Cheng et al. 2009, Weaver et al. 2011, Linton and Kozlowski 2008, Kumar et al. 2017), symmetrically 3,3-disubsitution can be very useful in particular examples. In this sense, there are two main approaches for achieving this last task (compounds 3); one of them is the cyclization of acylated anilides $\mathbf{1}$ (Figure 2, eq. a) and, the second (the most frequently used), the direct double alkylation onto $\mathbf{2}$, which is currently performed employing alkoxides or stronger bases (Figure 2, eq. b) (Scriven and Ramsden 2015).

In this context, deacylative alkylation (DaA) reaction emerged as an alternative to obtain this type of substitution (Mei et al. 2015, Kumar et al. 2017, Xie et al. 2016). The strategy of this transformation consists in the employment of the acetyl group as protecting, activating and leaving group for the alkylation sequence. It was recently

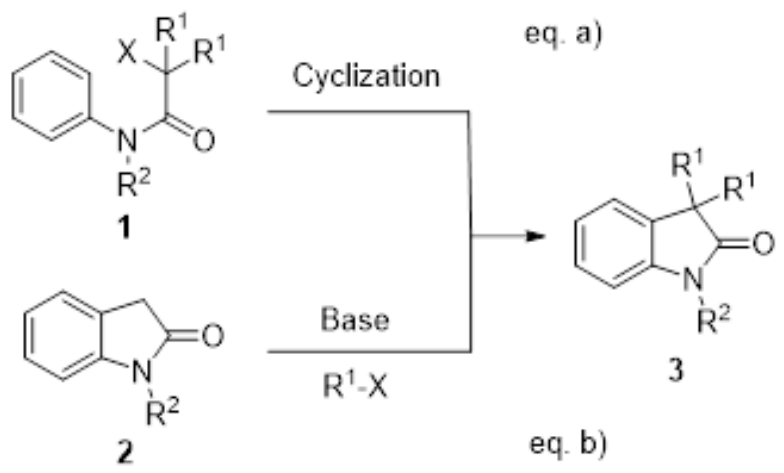

Figure 2 - Approaches to obtain 3,3-disubstituted 2-oxindoles. 
demonstrated by our group than the monoalkylation of 3-acetyl-2-oxindoles 4 (Ortega-Martínez et al. 2017) could be performed using alkyl halides and benzyltrimethylammonium hydroxide (Triton B) as base at room temperature in good yields. This methodology was applied to the synthesis of 1,3-dimethyl-2-oxindoles (Ortega-Martínez et al. 2017). Next, a deacylative alkylation (DaA) of the corresponding 3-acetyl-2-oxindole (5) with activated alkyl halides took place efficiently using LiOEt (Figure 3). In addition, conjugate addition with electron-deficient alkenes also was produced in the presence of Triton B. In both cases, the corresponding unsymmetrically 3,3-disubstituted 2-oxindoles 6 or 7 were isolated respectively (Figure 3) (Ortega-Martínez et al. 2017).

Continuing with our research looking for applications of this DaA for the synthesis of 3,3-dialkyloxindoles we describe here the synthesis of symmetrical 3,3-disubstituted analogues. We also have compared the results obtained through this DaA methodology versus the direct double alkylation of oxindole.

\section{EXPERIMENTAL SECTION}

\section{GENERAL}

Melting points were determined with a Marienfeld melting-point meter (MPM-H2) apparatus and are uncorrected. For flash chromatography, silica gel
$60(40-60 \mu \mathrm{m})$ was employed. The structurally most important peaks of the IR spectra (recorded using a Nicolet Avatar 320 FT-IR Spectrometer and JASCO FT/IR-4100 Fourier Transform Infrared Spectrometer) are listed and wave numbers are given in $\mathrm{cm}^{-1} .{ }^{1} \mathrm{H}$ NMR $(300$, or $400 \mathrm{MHz})$ and ${ }^{13} \mathrm{C}$ NMR (75 or $101 \mathrm{MHz}$ ) spectra were recorded with Bruker AV300 and Bruker AV400, respectively, with $\mathrm{CDCl}_{3}$ as solvent and TMS as internal standard for ${ }^{1} \mathrm{H}$ NMR spectra, and the chloroform signal for ${ }^{13} \mathrm{C}$ NMR spectra; chemical shifts are given in ppm. Low-resolution electron impact (GCEI) mass spectra were obtained at $70 \mathrm{eV}$ with an Agilent 6890N Network GC system and an Agilent 5973Network Mass Selective Detector. Highresolution mass spectra (GC-EI) were recorded with a QTOF Agilent 7200 instrument for the exact mass and Agilent 7890B for the GC. Analytical TLC was performed using ALUGRAM ${ }^{\circledR}$ Xtra SIL $\mathrm{G} / \mathrm{UV}_{254}$ silica gel plates, and the spots were detected under UV light $(\lambda=254 \mathrm{~nm})$. The synthesis of $N$-methyl-3-acetyl-2-oxindole was reproduced from the reported procedure (Ortega-Martínez et al. 2017).

\section{SYNTHESIS OF 3,3-DISUBSTITUTED OXINDOLES USING DAA. GENERAL PROCEDURE}

To a solution of $\mathrm{N}$-methyl-3-acetyl-2-oxindole 4a (57 $\mathrm{mg}, 0.3 \mathrm{mmol})$ and alkyl halide $(0.3 \mathrm{mmol})$ in THF ( $3 \mathrm{~mL})$ was added benzyltrimethylammonium

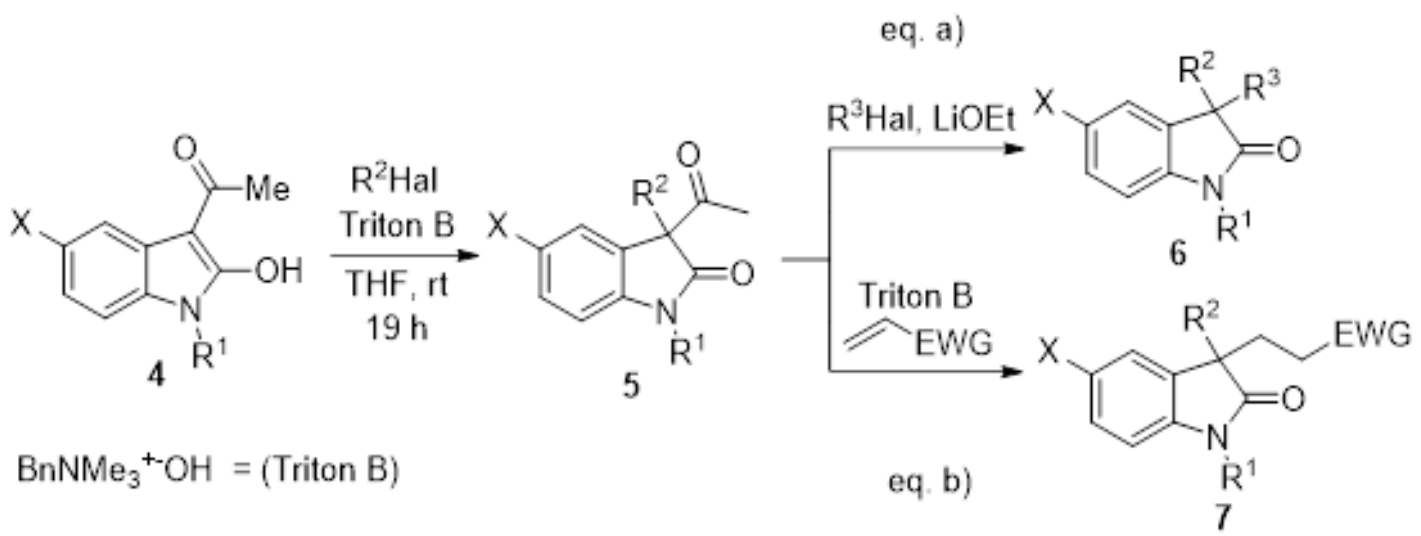

Figure 3 - Synthesis of unsimmetrically 3,3-disubstituted 2-oxindoles by our group. 
hydroxide (Triton B) in $\mathrm{MeOH}(40 \mathrm{wt} \%, 136 \mu \mathrm{L}$, $0.3 \mathrm{mmol}$ ) dropwise. The reaction was stirred at rt during 4-6 h. The reaction was controlled by gas chromatography until the conversion of $\mathbf{4 a}$ to 3-acetyl-3-alkyl-2-oxindole was $\geq 90 \%$. Then, alkyl halide $(0.45 \mathrm{mmol})$ and base $(0.45 \mathrm{mmol})$ was added to complete the double alkylation, allowing the reaction to proceed at room temperature overnight. $\mathrm{H}_{2} \mathrm{O}(10 \mathrm{~mL})$ was added, the mixture was extracted with EtOAc $(3 \times 10 \mathrm{~mL})$ and the combined organic layers were evaporated and dried over $\mathrm{MgSO}_{4}$. After evaporation of the solvents the residue was purified by flash chromatography (EtOAc/hexane).

1,3,3-Trimethylindolin-2-one (3a): colorless oil; $R_{F} 0.3$ (hexane/EtOAc 8.5:1.5); IR (neat) $v_{\max }$ 3053, 2970, 2924, 1704, $1613 \mathrm{~cm}^{-1}$; ${ }^{1} \mathrm{H}$ NMR (300 MHz) $\delta 7.27(1 \mathrm{H}, \mathrm{td}, J=7.7,1.3 \mathrm{~Hz}, \mathrm{ArH}), 7.21$ $(1 \mathrm{H}, \mathrm{d}, J=7.4 \mathrm{~Hz}, \mathrm{ArH}), 7.07(1 \mathrm{H}, \mathrm{td}, J=7.5$, $0.9 \mathrm{~Hz}, \mathrm{ArH}), 6.85(1 \mathrm{H}, \mathrm{d}, J=7.8 \mathrm{~Hz}, \mathrm{ArH}), 3.22$ $\left(3 \mathrm{H}, \mathrm{s}, \mathrm{NCH}_{3}\right), 1.37\left(6 \mathrm{H}, \mathrm{s}, 2 \times \mathrm{CH}_{3}\right) ;{ }^{13} \mathrm{C} \mathrm{NMR}$ (101 MHz) $\delta 181.5(\mathrm{CO}), 142.7(\mathrm{CH}), 135.9(\mathrm{CH})$, $127.8(\mathrm{CH}), 122.6(\mathrm{CH}), 122.4(\mathrm{CH}), 108.1(\mathrm{CH})$, $44.3(\mathrm{C}), 26.3\left(\mathrm{CH}_{3}\right), 24.5\left(2 \times \mathrm{CH}_{3}\right)$; LRMS (EI) $\mathrm{m} / z 175\left(\mathrm{M}^{+}, 66 \%\right), 161$ (11), 160 (100), 132 (20), 117 (14), 77(6); HRMS (ESI): calcd. for $\mathrm{C}_{11} \mathrm{H}_{13} \mathrm{NO}$ $[\mathrm{M}]^{+}$175.0997; found 175.0998 .

3,3-Diethyl-1-methylindolin-2-one (3b): colorless oil; $R_{F} 0.3$ (hexane/EtOAc 9:1); IR (neat) $v$ 2963, 2924, 2878, 2852, 1706, $1612 \mathrm{~cm}^{-1}$; ${ }^{1} \mathrm{H}$ NMR (300 MHz) $\delta$ 7.31-7.23 (1H, m, ArH), 7.17-7.04 $(2 \mathrm{H}, \mathrm{m}, \mathrm{ArH}), 6.84(1 \mathrm{H}, \mathrm{d}, J=7.7 \mathrm{~Hz}, \mathrm{ArH}), 3.22$ $\left(3 \mathrm{H}, \mathrm{s}, \mathrm{NCH}_{3}\right), 2.01-1.68\left(4 \mathrm{H}, \mathrm{m}, 2 \times \mathrm{CH}_{2}\right), 0.56$ $\left(6 \mathrm{H}, \mathrm{t}, J=7.4 \mathrm{~Hz}, 2 \times \mathrm{CH}_{3}\right) ;{ }^{13} \mathrm{C}$ NMR $(101 \mathrm{MHz})$ $\delta 180.2(\mathrm{CO}), 144.5(\mathrm{C}), 132.1(\mathrm{C}), 127.7(\mathrm{CH})$, $122.8(\mathrm{CH}), 122.5(\mathrm{CH}), 107.8(\mathrm{CH}), 54.5(\mathrm{C}), 30.8$ $\left(2 \times \mathrm{CH}_{2}\right), 26.1\left(\mathrm{CH}_{3}\right), 8.8\left(2 \times \mathrm{CH}_{3}\right)$; LRMS (EI) $\mathrm{m} / \mathrm{z} 203\left(\mathrm{M}^{+}, 72 \%\right), 204$ (10), 175 (51), 174 (100), 160 (20), 159 (12), 146 (66), 131 (19), 130 (25); HRMS (ESI): calcd. for $\mathrm{C}_{13} \mathrm{H}_{17} \mathrm{NO}[\mathrm{M}]^{+} 203.131$; found 203.1313.

3,3-Dibenzyl-1-methylindolin-2-one (3c): (Shi et al. 2014)
1-Methyl-3,3-bis(2-methylbenzyl)indolin2-one (3d): pale yellow solid; mp 102-104 ${ }^{\circ} \mathrm{C}$ (hexane/EtOAc) ; $R_{F} 0.3$ (hexane/EtOAc 9:1); IR (neat) $v$ 3058, 2963, 2923, 2857, 1709, $1611 \mathrm{~cm}^{-1}$; ${ }^{1} \mathrm{H}$ NMR $(400 \mathrm{MHz}) \delta 7.11(1 \mathrm{H}, \mathrm{td}, J=7.6,1.5 \mathrm{~Hz}$, ArH), 7.02-6.94 (4H, m, ArH), 6.92-6.80 (6H, m, ArH), $6.51(1 \mathrm{H}, \mathrm{d}, J=7.8 \mathrm{~Hz}, \mathrm{ArH}), 3.31(4 \mathrm{H}, \mathrm{s}, 2$ x $\left.\mathrm{CH}_{2}\right), 2.93\left(3 \mathrm{H}, \mathrm{s}, \mathrm{NCH}_{3}\right), 2.11\left(6 \mathrm{H}, \mathrm{s}, 2 \mathrm{x} \mathrm{CH}_{3}\right)$; ${ }^{13} \mathrm{C}$ NMR (101 MHz) $\delta 179.5$ (CO), $143.8(\mathrm{C})$, 137.19(C), 134.9 (C), 130.4 (C), $130.3(2 \times \mathrm{CH})$, $130.1(2 \times \mathrm{CH}), 128.0(\mathrm{CH}), 126.6(2 \times \mathrm{CH}), 125.2$ $(2 \times \mathrm{CH}), 124.8(\mathrm{CH}), 121.6(\mathrm{CH}), 107.7(\mathrm{CH})$, $55.2(2 \times \mathrm{C}), 39.2\left(2 \times \mathrm{CH}_{2}\right), 26.0\left(\mathrm{CH}_{3}\right), 20.3(2$ x $\mathrm{CH}_{3}$ ); LRMS (EI) $m / z 355\left(\mathrm{M}^{+}, 55 \%\right), 356$ (15), 251 (22), 250 (100), 222 (44), 207 (14), 159 (17), 105 (47); HRMS (ESI): calcd. for $\mathrm{C}_{25} \mathrm{H}_{25} \mathrm{NO}[\mathrm{M}]^{+}$ 355.1936; found 355.1943.

1 '-Methyl-1,3-dihydrospiro [indene-2,3'indolin]-2'-one (3e): (Frost et al. 2015)

3,3-Diallyl-1-methylindolin-2-one (3f): (Ortega-Martínez et al. 2017)

3,3-Dicinnamyl-1-methylindolin-2-one (3g): yellow oil; $R_{F} 0.3$ (hexane/EtOAc 9:1); IR (neat) $v$ 3053, 3024, 2926, 1701, $1614 \mathrm{~cm}^{-1}$; ${ }^{1} \mathrm{H}$ NMR (400 MHz) $\delta$ 7.32-7.12 (12H, m, ArH), 7.08 (1H, t, $J=$ $7.0 \mathrm{~Hz}, \mathrm{ArH}), 6.78(1 \mathrm{H}, \mathrm{d}, J=7.7 \mathrm{~Hz}, \mathrm{ArH}), 6.36$ $(2 \mathrm{H}, \mathrm{d}, J=15.8 \mathrm{~Hz}, 2 \times \mathrm{CH}), 5.86(2 \mathrm{H}, \mathrm{dt}, J=15.5$, $7.5 \mathrm{~Hz}, 2 \times \mathrm{CH}), 3.13\left(1 \mathrm{H}, \mathrm{s}, \mathrm{NCH}_{3}\right), 2.75(4 \mathrm{H}, \mathrm{d}$, $\left.J=7.4 \mathrm{~Hz}, 2 \times \mathrm{CH}_{2}\right) ;{ }^{13} \mathrm{C} \mathrm{NMR}(101 \mathrm{MHz}) \delta 179.0$ (CO), $143.8(\mathrm{C}), 137.4(2 \times \mathrm{C}), 133.9(2 \times \mathrm{CH})$, $131.4(\mathrm{C}), 128.5(4 \times \mathrm{CH}), 128.1(\mathrm{CH}), 127.3(2 \times$ $\mathrm{CH}), 126.3(4 \times \mathrm{CH}), 124.1(2 \times \mathrm{CH}), 123.6(\mathrm{CH})$, $122.5(\mathrm{CH}), 108.1(\mathrm{CH}), 53.4(\mathrm{C}), 40.4\left(2 \mathrm{x} \mathrm{CH}_{2}\right)$, $26.3\left(\mathrm{CH}_{3}\right)$; LRMS (EI) $\mathrm{m} / z 379\left(\mathrm{M}^{+}, 32 \%\right) 380$ (12), 288 (12), 281 (17), 262 (43), 261 (14), 234 (11), 208 (11), 207 (54), 147 (45), 146 (23), 118 (23), 117 (100), 116 (10), 115 (34), 91 (14); HRMS (ESI): calcd. for $\mathrm{C}_{27} \mathrm{H}_{25} \mathrm{NO}[\mathrm{M}]^{+}$379.1936; found 379.1939 .

3,3-Bis((E)-3,7-dimethylocta-2,6-dien-1-yl)1-methylindolin-2-one (3h): yellow oil; $R_{F} 0.2$ (hexane/EtOAc 9.5:0.5); IR (neat) v 2965, 2921, 
2855, 1717, $1612 \mathrm{~cm}^{-1} ;{ }^{1} \mathrm{H}$ NMR (300 MHz) $\delta$ 7.26-7.18 (2H, m, ArH), 7.01 (1H, td, $J=7.5,0.9$ $\mathrm{Hz}, \mathrm{ArH}), 6.77$ (1H, d, $J=7.7 \mathrm{~Hz}, \mathrm{ArH}), 4.99-4.88$ $(2 \mathrm{H}, \mathrm{m}, 2 \times \mathrm{CH}), 4.80(2 \mathrm{H}, \mathrm{t}, J=7.0 \mathrm{~Hz}, 2 \times \mathrm{CH})$, $3.16\left(3 \mathrm{H}, \mathrm{s}, \mathrm{NCH}_{3}\right), 2.53(4 \mathrm{H}, \mathrm{d}, J=7.5 \mathrm{~Hz}, 2 \mathrm{x}$ $\left.\mathrm{CH}_{2}\right), 1.90-1.65\left(8 \mathrm{H}, \mathrm{m}, 4\right.$ x $\left.\mathrm{CH}_{2}\right), 1.63(6 \mathrm{H}, \mathrm{s}, 2 \mathrm{x}$ $\left.\mathrm{CH}_{3}\right), 1.53\left(6 \mathrm{H}, \mathrm{s}, 2 \times \mathrm{CH}_{3}\right), 1.51\left(6 \mathrm{H}, \mathrm{s}, 2 \times \mathrm{CH}_{3}\right)$; ${ }^{13} \mathrm{C}$ NMR $(75 \mathrm{MHz}) \delta 180.0(\mathrm{CO}), 144.0(\mathrm{C}), 138.6$ $(2 \times \mathrm{C}), 132.3(\mathrm{C}), 131.4(2 \times \mathrm{C}), 127.6(\mathrm{CH}), 124.3$ $(2 \times \mathrm{CH}), 123.5(\mathrm{CH}), 122.0(\mathrm{CH}), 118.3(2 \times \mathrm{CH})$, $107.5(\mathrm{CH}), 53.4(\mathrm{C}), 39.9\left(2 \mathrm{x} \mathrm{CH}_{2}\right), 35.3(2 \mathrm{x}$ $\left.\mathrm{CH}_{2}\right), 26.8\left(2 \times \mathrm{CH}_{2}\right), 26.1\left(\mathrm{CH}_{3}\right), 25.8\left(2 \times \mathrm{CH}_{3}\right)$, $17.7\left(2 \mathrm{x} \mathrm{CH}_{3}\right), 16.5\left(2 \times \mathrm{CH}_{3}\right)$; LRMS (EI) $m / z 419$ $\left(\mathrm{M}^{+}, 3 \%\right), 283$ (35), 214 (10), 198 (18), 161 (11), 160 (91), 159 (100), 147 (24), 146 (14), 81 (12), 69 (39); HRMS (ESI): calcd. for $\mathrm{C}_{29} \mathrm{H}_{41} \mathrm{NO}[\mathrm{M}]^{+}$ 419.3188; found 419.3191 .

Dimethyl 4,4'-(1-methyl-2-oxoindoline3,3-diyl)bis(but-2-enoate) (3i): pale oil; $R_{F} 0.25$ (hexane/EtOAc 7:3); IR (neat) v 2950, 1707, 1611 $\mathrm{cm}^{-1}$; ${ }^{1} \mathrm{H}$ NMR $(300 \mathrm{MHz}) \delta 7.30(1 \mathrm{H}, \mathrm{td}, J=$ 7.7, 1.3 Hz, ArH), 7.21-7.17 (1H, m, ArH), 7.09 $(1 \mathrm{H}, \mathrm{td}, J=7.5,0.9 \mathrm{~Hz}, \mathrm{ArH}), 6.85(1 \mathrm{H}, \mathrm{d}, J=$ $7.8 \mathrm{~Hz}, \mathrm{ArH}), 6.54(2 \mathrm{H}, \mathrm{dt}, J=15.4,7.6 \mathrm{~Hz}, 2 \mathrm{x}$ $\mathrm{CH}), 5.79(2 \mathrm{H}, \mathrm{d}, J=15.6 \mathrm{~Hz}, 2 \mathrm{x} \mathrm{CH}), 3.65(6 \mathrm{H}$, $\left.\mathrm{s}, 2 \times \mathrm{OCH}_{3}\right), 3.19\left(3 \mathrm{H}, \mathrm{s}, \mathrm{NCH}_{3}\right), 2.70(4 \mathrm{H}, \mathrm{dd}$, $\left.J=7.7,1.3 \mathrm{~Hz}, 2 \times \mathrm{CH}_{2}\right) ;{ }^{13} \mathrm{C} \mathrm{NMR}(75 \mathrm{MHz}) \delta$ 177.55 (CO), 166.3 ( 2 x CO), 143.4 (C), 142.1 ( 2 x $\mathrm{CH}), 129.6(\mathrm{C}), 128.9(\mathrm{CH}), 124.9(2 \times \mathrm{CH}), 123.3$ $(\mathrm{CH}), 123.0(\mathrm{CH}), 108.7(\mathrm{CH}), 51.7(\mathrm{C}), 51.6(2 \mathrm{x}$ $\left.\mathrm{CH}_{3}\right), 39.5\left(2 \times \mathrm{CH}_{2}\right), 26.4\left(\mathrm{CH}_{3}\right)$; LRMS (EI) $\mathrm{m} / \mathrm{z}$ $343\left(\mathrm{M}^{+}, 30 \%\right) 312$ (14), 245 (14), 244 (88), 212 (46), 185 (23), 184 (100); HRMS (ESI): calcd. for $\mathrm{C}_{19} \mathrm{H}_{21} \mathrm{NO}_{5}[\mathrm{M}]^{+}$343.142; found 343.1424.

Diethyl 4,4'-(1-methyl-2-oxoindoline-3,3-diyl) (2E,2'E)-bis(but-2-enoate) (3j): brown oil; $R_{F} 0.3$ (hexane/EtOAc); IR (neat) $v 2981,2937,1716$, $1655 \mathrm{~cm}^{-1}$; ${ }^{1} \mathrm{H}$ NMR $(300 \mathrm{MHz}) \delta 7.30(1 \mathrm{H}, \mathrm{t}, J=$ 7.7 Hz, ArH), 7.16-7.07 (2H, m, ArH), 6.84 (1H, d, $J=7.8 \mathrm{~Hz}, \mathrm{ArH}), 6.53(2 \mathrm{H}, \mathrm{dt}, J=15.4,7.6 \mathrm{~Hz}, 2 \mathrm{x}$ $\mathrm{CH}), 5.78(2 \mathrm{H}, \mathrm{d}, J=15.5 \mathrm{~Hz}, 2 \times \mathrm{CH}), 4.34-3.95$ $\left(4 \mathrm{H}, \mathrm{m}, 2 \times \mathrm{CH}_{2}\right), 3.19\left(3 \mathrm{H}, \mathrm{s}, \mathrm{NCH}_{3}\right), 2.70(4 \mathrm{H}, \mathrm{dd}$,
$\left.J=7.7,1.3 \mathrm{~Hz}, 2 \times \mathrm{CH}_{2}\right), 1.26(6 \mathrm{H}, \mathrm{dt}, J=22.4,7.1$ $\left.\mathrm{Hz}, 2 \times \mathrm{CH}_{3}\right) ;{ }^{13} \mathrm{C}$ NMR $(101 \mathrm{MHz}) \delta 177.6(\mathrm{CO})$, 165.9 ( 2 x CO), $143.4(\mathrm{C}), 141.7(2 \times \mathrm{CH}), 129.6$ $(\mathrm{CH}), 128.8(\mathrm{C}), 125.3(2 \times \mathrm{CH}), 123.3(\mathrm{CH}), 123.0$ $(\mathrm{CH}), 108.6(\mathrm{CH}), 60.4\left(2 \times \mathrm{CH}_{2}\right), 51.7(\mathrm{C}), 39.4(2$ $\left.\mathrm{x} \mathrm{CH}_{2}\right), 26.3\left(\mathrm{CH}_{3}\right), 14.2\left(2 \mathrm{x} \mathrm{CH}_{3}\right) ; \mathrm{LRMS}(\mathrm{EI}) \mathrm{m} / \mathrm{z}$ $371\left(\mathrm{M}^{+}, 13 \%\right), 258$ (54), 230 (10), 212 (24), 186 (14), 185 (27), 184 (100), 158 (11), 156 (14), 147 (11), 144 (12), 130 (10), 128 (13), 77 (13); HRMS (ESI): calcd. for $\mathrm{C}_{21} \mathrm{H}_{25} \mathrm{NO}_{5}[\mathrm{M}]^{+}$371.1733; found 371.1728 .

1-Methyl-3,3-di(prop-2-yn-1-yl)indolin-2-one (3k): (Zhou et al. 2013).

Dimethyl 2,2'-(1-methyl-2-oxoindoline-3,3diyl)diacetate (31): yellow wax; $R_{F} 0.3$ (hexane/ EtOAc 6.5:3.5); IR (neat) $v 2997,2950,1711,1612$ $\mathrm{cm}^{-1} ;{ }^{1} \mathrm{H}$ NMR $(300 \mathrm{MHz}) \delta 7.30(2 \mathrm{H}, \mathrm{t}, J=7.0 \mathrm{~Hz}$, ArH), 7.03 (1H, t, $J=7.6 \mathrm{~Hz}, \operatorname{ArH}), 6.87(1 \mathrm{H}, \mathrm{d}, J$ $=8.0 \mathrm{~Hz}, \mathrm{ArH}), 3.50\left(6 \mathrm{H}, \mathrm{s}, 2 \times \mathrm{OCH}_{3}\right), 3.28(3 \mathrm{H}$, $\left.\mathrm{s}, \mathrm{NCH}_{3}\right), 3.06(2 \mathrm{H}, \mathrm{d}, J=16.2 \mathrm{~Hz}, 2 \times \mathrm{CHH}), 2.87$ $(2 \mathrm{H}, \mathrm{d}, J=16.2 \mathrm{~Hz}, 2 \times \mathrm{CH} H) ;{ }^{13} \mathrm{C} \mathrm{NMR}(75 \mathrm{MHz})$

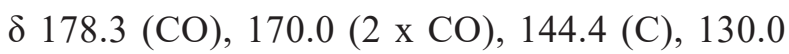
(C), $128.8(\mathrm{CH}), 123.4(\mathrm{CH}), 122.5(\mathrm{CH}), 108.3$ (CH), $51.8\left(2 \times \mathrm{CH}_{3}\right), 46.9(\mathrm{C}), 40.5\left(2 \times \mathrm{CH}_{2}\right)$, $26.6\left(\mathrm{CH}_{3}\right)$; LRMS (EI) $\mathrm{m} / z 291\left(\mathrm{M}^{+}, 100 \%\right), 292$ (17), 232 (29), 218 (13), 200 (13), 186 (21), 176 (51), 174 (21), 159 (43), 144 (16), 130 (25); HRMS (ESI): calcd. for $\mathrm{C}_{15} \mathrm{H}_{17} \mathrm{NO}_{5}[\mathrm{M}]^{+}$291.1107; found 291.1103.

2,2'-(1-Methyl-2-oxoindoline-3,3-diyl) diacetonitrile (3m): orange solid; $\mathrm{mp} 104-106{ }^{\circ} \mathrm{C}$; $R_{F} 0.3$ (hexane/EtOAc 6.5:3.5); IR (neat) v 2964, 2932, 1705, $1615 \mathrm{~cm}^{-1} ;{ }^{1} \mathrm{H}$ NMR (400 MHz) $\delta 7.58$ $(1 \mathrm{H}, \mathrm{d}, J=8.0 \mathrm{~Hz}, \mathrm{ArH}), 7.46(1 \mathrm{H}, \mathrm{td}, J=7.8,1.2$ $\mathrm{Hz}, \mathrm{ArH}), 7.22(1 \mathrm{H}, \mathrm{td}, J=7.7,0.9 \mathrm{~Hz}, \mathrm{ArH}), 6.98$ $(1 \mathrm{H}, \mathrm{d}, J=7.9 \mathrm{~Hz}, \mathrm{ArH}), 3.29\left(3 \mathrm{H}, \mathrm{s}, \mathrm{NCH}_{3}\right), 3.02$ $(2 \mathrm{H}, \mathrm{d}, J=16.7 \mathrm{~Hz}, 2 \times \mathrm{CHH}), 2.82(2 \mathrm{H}, \mathrm{d}, J=$ $16.7 \mathrm{~Hz}, 2$ x CHH); ${ }^{13} \mathrm{C}$ NMR (101 MHz) $\delta 173.9$ (CO), $143.2(\mathrm{C}), 130.8(\mathrm{CH}), 124.0(\mathrm{CH}), 126.4$ (C), $123.6(\mathrm{CH}), 115.1(2 \times \mathrm{CN}), 109.5(\mathrm{CH}), 45.8$ (C), $26.9\left(\mathrm{CH}_{3}\right), 24.8\left(2 \times \mathrm{CH}_{2}\right)$; LRMS (EI) $\mathrm{m} / \mathrm{z}$ $225\left(\mathrm{M}^{+}, 33 \%\right), 186$ (13), 185 (100), 155 (5), 142 
(6), 128 (7); HRMS (ESI): calcd. for $\mathrm{C}_{13} \mathrm{H}_{11} \mathrm{~N}_{3} \mathrm{O}$ $[\mathrm{M}]^{+}$225.0902; found 225.0907

SYNTHESIS OF SPIROOXINDOLE 9 THROUGH A RUTHENIUM CATALYZED METATHESIS REACTION

To a solution of $2^{\text {nd }}$ generation Grubbs catalyst $(0.002 \mathrm{mmol}, 1.7 \mathrm{mg})$ in dry dichloromethane $(20$ $\mathrm{mL})$ compound $\mathbf{3 f}(0.2 \mathrm{mmol}, 45 \mathrm{mg})$ was added. The mixture was stirred during $1.5 \mathrm{~h}$ at $42^{\circ} \mathrm{C}$ under $\mathrm{Ar}$ atmosphere. The solution was filtered off through a short plug of celite. Finally, the solution was concentrated (15 Torr) and the residue was purified by column chromatography (hexane/ EtOAc) obtaining 1'-Methylspiro[cyclopentane1,3'-indolin]-3-en-2'-one (9) (Kattela et al. 2017).

\section{RESULTS AND DISCUSSION}

The reaction was initially optimized using different bases (Ortega-Martínez et al. 2017) concluding that the most appropriate base was benzyltrimethylammonium hydroxide (Triton B) in THF as solvent at room temperature. The direct transformation employing 2.5 equiv. of both organic halide and base was not useful due to the formation of monoalkylated deacylated 2-oxindoles. For this reason, the double alkylation was performed in a one pot sequential process. On it, the first step consisted in the addition of the electrophile and the base (1 equiv. each reagent) in this order. After $4 \mathrm{~h}$ another addition of the same electrophile and base (1.5 equiv. each reagent) took place in order to complete the double alkylation mediated by the deacylative process, allowing the reaction to proceed at room temperature overnight. Compounds $\mathbf{3}$ were finally purified and isolated after column chromatography (flash silica gel) in very good to moderate yields (Figure 4, and Table I). When alkyl iodides were employed the chemical yields of products $\mathbf{3 a}$ and $\mathbf{3 b}$ were 51 and $33 \%$, respectively (Table I, entries 1 and 2). This low conversion was caused by the relative low reactivity towards these alkyl

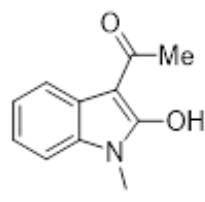

1. $\mathrm{RHal}$ (1 equiv.)

$\operatorname{THF}(0.1 \mathrm{M})$, rt, $4 \mathrm{~h}$

2. $\mathrm{RHal}$ ( 1.5 equiv)

$\operatorname{THF}(0.1 \mathrm{M}), \mathrm{rt}_{\mathrm{t}} 19 \mathrm{~h}$

4a, $R^{1}=M e, X=H$ $\mathrm{BnNMe}_{3}{ }^{+-} \mathrm{OH}$ (1 equiv.)

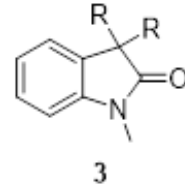

Figure 4 - DaA in the generation of 3,3-disubstituted oxindole 3.

halides, and with the competition with the easy oxidation at the benzylic position. Thus, a $88: 12$ mixture of 3a:3-hydroxy-1,3-dimethyloxindole and a 61:39 mixture of $\mathbf{3 b}$ :3-hydroxy-3-ethyl-1methyloxindole was observed by ${ }^{1} \mathrm{H}$ NMR (crude product). More activated halides such as benzylic bromides appearing in the three next entries of Table I furnished higher chemical yields in a range of 69$85 \%$. Bisbenzylic unit was efficiently introduced providing spiranic oxindole derivative $\mathbf{3 e}$, whose skeleton is present in antitumor agents (Yang et al. 2016) and aldose reductase inhibitors (Howard et al. 1992). Allylic bromides such as allyl bromide and cinnamyl bromide also gave good conversions and yields 65 and $84 \%$, respectively and no other byproduct was identified by ${ }^{1} \mathrm{H}$ NMR of the crude product (Table I, entries 6 and 7). The employment of geranyl bromide furnished a complex reaction mixture, which, after flash chromatography, allowed to isolate an inseparable 65:35 mixture of $\mathbf{3 h}$ together with its corresponding deacylated 3-monoalkylated compound (Table I, entry 8). Technical methyl bromocrotonate and ethyl $E$-bromocrotonate gave similar results of $\mathbf{3 i}$ and $\mathbf{3 j}$ after this transformation, the crude compound $\mathbf{3 i}$ being much more complex due to the presence of $Z$ - and $E$-steroisomers (Table I, entries 9 and 10). Another three halides with $\pi$-extended conjugation were tested. Propargyl bromide gave a 58\% yield of the disubstituted product $\mathbf{3 k}$ (Table I, entry 11), methyl bromoacetate afforded a similar $61 \%$ yield (Table I, entry 12) and finally, bromoacetonitrile furnished the best chemical $82 \%$ yield of this 
TABLE I

Synthesis of 3,3-disubstituted-2-oxindoles 3 using a DaA of 4a.

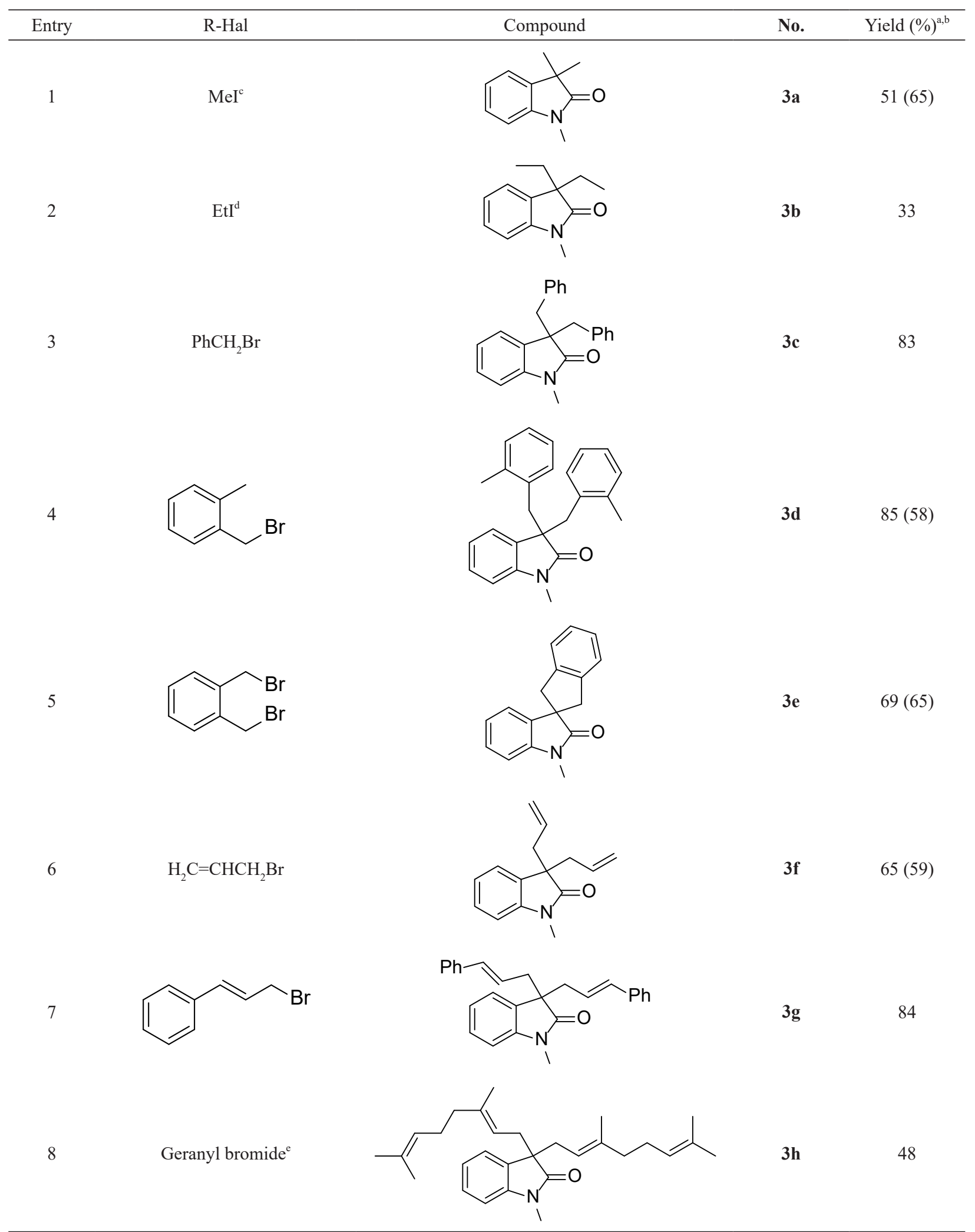


TABLE I (continuation)

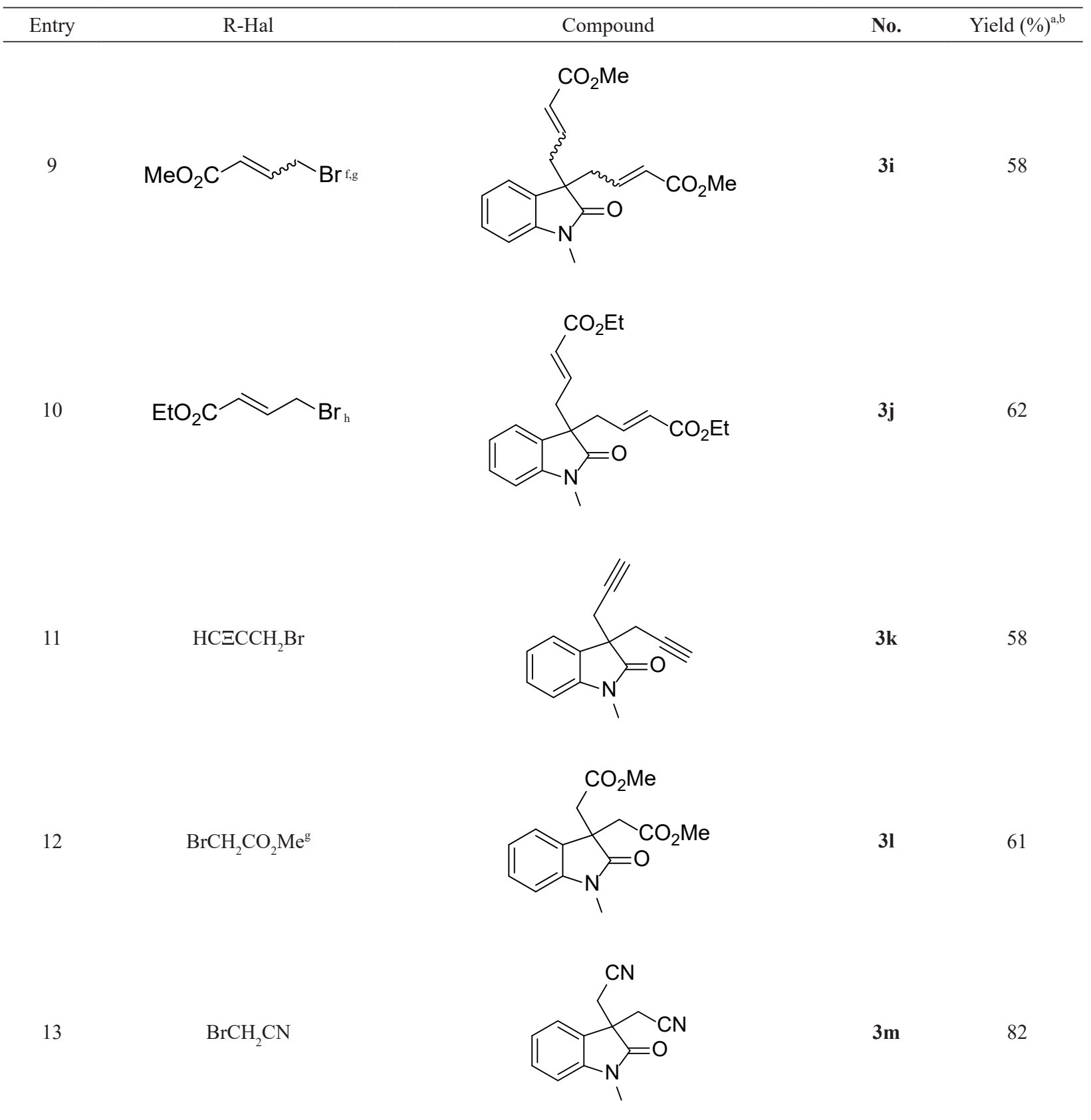

${ }^{a}$ Isolated yield after column chromatography (flash silica).

${ }^{\mathrm{b}}$ In brackets, yields obtained from a direct dialkylation of $\mathrm{N}$-methyloxindole.

${ }^{\mathrm{c}}$ A 88:12 mixture of 3a:3-hydroxy-1,3-dimethyloxindole was observed by NMR (crude product).

${ }^{\mathrm{d}}$ A 61:39 mixture of 3b:3-hydroxy-3-ethyl-1-methyloxindole was observed by NMR (crude product).

${ }^{\mathrm{e}}$ This compound was obtained as an inseparable 65:35 mixture of $\mathbf{3 h}$ and its corresponding deacylated 3-monoalkylated compound.

${ }^{\mathrm{f}}$ This compound was obtained as 81:19 mixture of $\mathbf{3 i}$ and its corresponding deacylated 3-monoalkylated compound, but $\mathbf{3 i}$ could be finally separated.

g In this example, the sequential process was performed using: 1. RHal (1 equiv.) and Triton B (1 equiv.) at rt for $4 \mathrm{~h}$; 2 . RHal (1 equiv.) and Triton B (1 equiv.) at rt for 19 h; 3. RHal (0.5 equiv.) and Triton B (0.5 equiv.) at rt for $19 \mathrm{~h}$.

${ }^{\mathrm{h}}$ This compound was obtained as an inseparable 65:35 mixture of $\mathbf{3} \mathbf{j}$ and its corresponding deacylated 3-monoalkylated compound. 
series (Table I, entry 13). In entries 9 and 12 of the Table I a different protocol was employed in order to achieve higher yields. Thus, the addition of the total amount of reagents was performed adding RHal (1 equiv.) and Triton B (1 equiv.) at $\mathrm{rt}$ for 4 $\mathrm{h}$, followed by de addition of RHal (1 equiv.) and Triton B (1 equiv.) at $\mathrm{rt}$ for $19 \mathrm{~h}$ and, finally RHal ( 0.5 equiv.) and Triton $B(0.5$ equiv.) at $\mathrm{rt}$ for $19 \mathrm{~h}$ completed the sequence.

In some examples depicted in Table I, a comparison of this deacylative route with the direct dialkylation of oxindole $\mathbf{8}$ with excess of both Triton B and the corresponding halide (Figure 5) was made. The direct alkylation was much more efficient when methyl iodide was tested ( $65 \%$ versus $51 \%$, see Table I, entry 1 ). However, the yield of compound $\mathbf{3 d}$, using the DaA route, increased manifold ( $85 \%$ versus $58 \%$, Table I, entry 4). 2-(Bromomethyl)benzyl bromide and allyl bromide gave very similar results using both reaction sequences such as it was depicted in Table I (entries 5 and 6) but the crude of the DaA mediated reactions were cleaner than the corresponding ones for the direct dialkylation way.

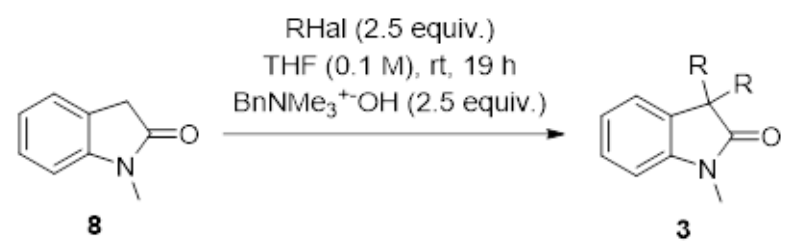

Figure 5 - Synthesis of 3,3-disubstituted oxindole 3 from $\mathrm{N}$-methyloxindole (8).

The application of $\mathbf{3 f}$ for the synthesis of other structurally complex containing this oxindole unit was envisaged. The metathesis reaction employing the $2^{\text {nd }}$ generation Grubbs' catalyst produced spirocompound 9 in quantitative yield after $1.5 \mathrm{~h}$ under refluxing dichloromethane (Figure 6). The presence of the residual carbon-carbon double bond would allow the access to core framework of natural compounds citrinadins A and B (Bian et al. 2014) as well as the preparation of families of mentioned antitumor spiroxindole derivatives (see above).

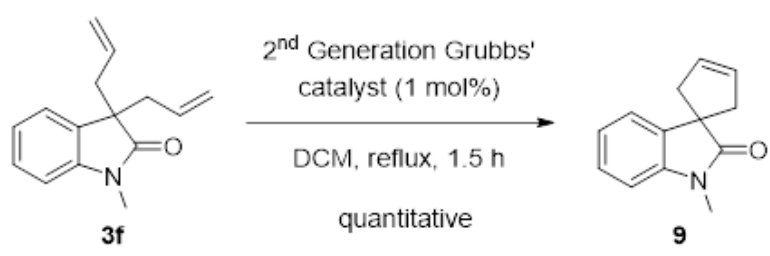

Figure 6 - Synthesis of spirooxindole 9 through a rutheniumcatalyzed metathesis reaction.

\section{CONCLUSIONS}

The sequential one-pot monoalkylation of 3-acetyl-1-methyl-2-oxindole, followed by a DaAsecond alkylation is an alternative way to obtain symmetrical 3,3-disubstituted oxindole derivatives. The process competes with the direct dialkylation of $\mathrm{N}$-methyloxindole because, in many cases, yields are higher and the crude materials cleaner, which are two very strong points in favor of this strategy. Some compounds obtained in this work (specifically $\mathbf{3 e}$ and $\mathbf{9}$ ) were suitable candidates to access interesting antitumor agents and natural compounds.

\section{ACKNOWLEDGMENTS}

Financial support was provided by the Spanish Ministerio de Economía y Competitividad (MINECO) (projects CTQ2013-43446-P and CTQ2014-51912REDC), the Spanish Ministerio de Economía, Industria y Competitividad, Agencia Estatal de Investigación (AEI) and Fondo Europeo de Desarrollo Regional (FEDER, EU) (projects CTQ201676782-P and CTQ2016-81797-REDC), by the Generalitat Valenciana (PROMETEO2009/039 and PROMETEOII/ 2014/017) and by the University of Alicante. Aitor Ortega-Martínez thanks MINECO for a predoctoral fellowship. 


\section{REFERENCES}

ALTMAN RA, HYDE AM, HUANG X AND BUCHWALD SL. 2008. Orthogonal Pd- and Cu-based catalyst systems for C- and N-arylation of oxindoles. J Am Chem Soc 130: 9613-9620.

ASHIMORI A, MATSUURA T, OVERMAN LE AND POON DJ. 1993. Catalytic asymmetric synthesis of either enantiomer of physostigmine. Formation of quaternary carbon centers with high enantioselection by intramolecular Heck reactions of (Z)-2-butenanilides. J Org Chem 58: 6949-6951.

BIAN Z, MARVIN CC, PETTERSSON M AND MARTIN SF. 2014. Enantioselective total syntheses of citrinadins A and B. Stereochemical revision of their assigned structures. J Am Chem Soc 136: 14184-14192.

CAO ZY, WANG YH, ZENG YP AND ZHOU J. 2014. Catalytic asymmetric synthesis of 3,3-disubstituted oxindoles: diazooxindole joins the field. Tetrahedron Lett 55: 2571-2754.

CHENG L, LIU L, JIA H, WANG D AND CHEN YJJ. 2009. Enantioselective organocatalytic anti-Mannich-type reaction of $\mathrm{N}$-unprotected 3-substituted 2-oxindoles with aromatic N-Ts-aldimines. Org Chem 74: 4650-4653.

DALPOZZO R, BARTOLI G AND BENCIVENNI G. 2012. Recent advances in organocatalytic methods for the synthesis of disubstituted 2- and 3-indolinones. Chem Soc Rev 41: 7247-7290.

DAVID N, PASCERI R, KITSON RRA, PRADAL A AND MOODY CJ. 2016. Formal total synthesis of diazonamide A by indole oxidative rearrangement. Chem Eur J 22: 10867-10876.

DONDAS HA, RETAMOSA MG AND SANSANO JM. 2017. Current trends towards the synthesis of bioactive heterocycles and natural products using 1,3-dipolar cycloadditions (1,3-DC) with azomethine ylides. Synthesis 49: 2819-2851.

FONSECA GO AND COOK JM. 2016. Modern methods for total synthesis of important oxindole alkaloids. Org Chem Ins 6: 1-55.

FROST JR, HUBER SM, BREITENLECHNER S, BANNWARTH C AND BACH T. 2015. EnantiotoposSelective C-H Oxygenation Catalyzed by a Supramolecular Ruthenium Complex. Angew Chem Int Ed 54: 691-695.

GALLIFORD CV AND SCHEIDT KA. 2007. Pyrrolidinylspirooxindole natural products as inspirations for the development of potential therapeutic agents. Angew Chem Int Ed 46: 8748-8758.

GHOSH S, DE S, KAKDE BN, BHUNIA S, ADHIKARY A AND BISAI A. 2012. Intramolecular dehydrogenative coupling of sp2 $\mathrm{C}-\mathrm{H}$ and sp3 $\mathrm{C}-\mathrm{H}$ Bonds: An expeditious route to 2-oxindoles. Org Lett 14: 5864-5867.
GUPTA AK, BHARADWAJ M, KUMAR A AND MEHROTRA R. 2017. Spiro-oxindoles as a promising class of small molecule inhibitors of p53-MDM2 interaction useful in targeted cancer therapy. Top Curr Chem 375: 1-25.

HE R, DING C AND MARUOKA K. 2009. Phosphonium salts as chiral phase-transfer catalysts: asymmetric Michael and Mannich reactions of 3-aryloxindoles. Angew Chem Int Ed 48: 4559-4561.

HOWARD HR, SARGES R, SIEGEL TW AND BEYER TA. 1992. Synthesis and aldose reductase inhibitory activity of substituted $2(1 \mathrm{H})$-benzimidazolone- and oxindole-1-acetic acids. Eur J Med Chem 27: 779-789.

HUANG A, KODANKO J AND OVERMAN LE. 2004. Asymmetric synthesis of pyrrolidinoindolines. Application for the practical total synthesis of (-)-phenserine. J Am Chem Soc 126: 14043-14053.

JIA YX AND KUNDIG EP. 2009. Oxindole synthesis by direct coupling of Csp2-H and Csp3-H Centers. Angew Chem Int Ed 48: 1636-1639.

KATTELA S, HEERDT G AND CORREIA CRD. 2017. Non-covalent carbonyl-directed Heck-Matsuda desymmetrizations: synthesis of cyclopentene-fused spirooxindoles, spirolactones, and spirolactams. Adv Synth Cat 359: 260-267.

KAUR M, SINGH M, CHADHA N AND SILAKARI O. 2016. Oxindole: A chemical prism carrying plethora of therapeutic benefits. Eur J Med Chem 123: 858-894.

KUMAR N, DAS MK, GHOSH S AND BISAI A. 2017. Development of catalytic deacylative alkylations (DaA) of 3-acyl-2-oxindoles: total synthesis of meso-chimonanthine and related alkaloids. Chem Commun 53: 2170-2173.

KUNDIG EP, SEIDEL TM, JIA YX AND BERNARDINELLI G. 2007. Bulky chiral carbene ligands and their application in the palladium-catalyzed asymmetric intramolecular $\alpha$-arylation of amides. Angew Chem Int Ed 46: 8484-8487.

LINTON EC AND KOZLOWSKI MC. 2008. Catalytic enantioselective Meerwein-Eschenmoser Claisen rearrangement: asymmetric synthesis of allyl oxindoles. J Am Chem Soc 130: 16162-16163.

MARSDEN SP, WATSONAND EL AND RAW SA. 2008. Facile and general synthesis of quaternary 3-aminooxindoles. Org Lett 10: 2905-2908.

MATSUURA T, OVERMAN LE AND POON DJ. 1998. Catalytic Asymmetric Synthesis of Either Enantiomer of the Calabar Alkaloids Physostigmine and Physovenine. J Am Chem Soc 120: 6500-6503.

MEI H, XIE C, ACEÑA JL, SOLOSHONOK VA, RÖSCHENTHALER GV AND HAN J. 2015. Recent progress in the in situ detrifluoroacetylative generation of fluoro enolates and their reactions with electrophiles. Eur J Org Chem 6401-6412. 
ORTEGA-MARTÍNEZ A, MOLINA C, MORENOCABRERIZO C, SANSANO JM AND NÁJERA C. 2017. Synthesis of 3,3-disubstituted 2-oxindoles by deacylative alkylation of 3-acetyl-2-oxindoles. Synthesis 49: 5203-5210.

PERRY A AND TAYLOR RJK. 2009. Oxindole synthesis by direct $\mathrm{C}-\mathrm{H}, \mathrm{Ar}-\mathrm{H}$ coupling. Chem Commun, p. 32493251.

RUCK TR, HUFFMAN MA, KIM MM, SHEVLIN M, KANDUR WV AND DAVIES IW. 2008. Palladiumcatalyzed tandem Heck reaction/C-H functionalization-preparation of spiro-indane-oxindoles. Angew Chem Int Ed 47: 4711-4714.

SARASWAT P, JEYABALAN G, HASSAN MZ, RAHMAN MU AND NYOLA NK. 2016. Review of synthesis and various biological activities of spiro heterocyclic compounds comprising oxindole and pyrrolidine moieties. Synth Commun 46: 1643-1664.

SCRIVEN EFV AND RAMSDEN CA (Eds). 2015. Advances in heterocyclic chemistry. Academic Press: Oxford, vol. 116.

SHAUGHNESSY KH, HAMANN BC AND HARTWIG JF. 1998. Palladium-catalyzed inter- and intramolecular $\alpha$-arylation of amides. Application of intramolecular amide arylation to the synthesis of oxindoles. J Org Chem 63: 6546-6553.

SHEN K, LIU X AND FENG X. 2012. Recent progress in enantioselective synthesis of $\mathrm{C} 3$-functionalized oxindoles: rare earth metals take action. Chem Sci 3: 327-334.

SHI L, WANG Y, YANG H AND FU H. 2014. Coppercatalyzed bis-arylations of alkenes leading to oxindole derivatives. Org Biomol Chem 12: 4070-4073.

SINGH GS AND DESTA ZY. 2012. Isatins as privileged molecules in design and synthesis of spiro-fused cyclic frameworks. Chem Rev 112: 6104-6155.

TIAN X, JIANG K, PENG J, DU W AND CHEN YC. 2008. Organocatalytic stereoselective Mannich reaction of 3-substituted oxindoles. Org Lett 10: 3583-3586.

TROST BM AND BRENNAN MK. 2006. Palladium asymmetric allylic alkylation of prochiral nucleophiles: Horsfiline. Org Lett 8: 2027-2030.
TROST BM, MALHOTRA S AND CHAN WH. 2011. Exercising regiocontrol in palladium-catalyzed asymmetric prenylations and geranylation: unifying strategy toward flustramines A and B. J Am Chem Soc 133: 7328-7331.

TROST BM AND ZHANG YJ. 2006. Molybdenum-catalyzed asymmetric allylation of 3-alkyloxindoles: Application to the formal total synthesis of (-)-physostigmine. J Am Chem Soc 128: 4590-5080.

WEAVER JD, RECIO III A, GRENNING AJ AND TUNGE JA. 2011. Transition metal-catalyzed decarboxylative allylation and benzylation reactions. Chem Rev 111: 18461913.

XIE C, ZHANG L, SHA W, SOLOSHONOK VA, HAN J AND PAN Y. 2016. Detrifluoroacetylative in situ generation of free 3-fluoroindolin-2-one-derived tertiary enolates: design, synthesis, and assessment of reactivity toward asymmetric Mannich reactions. Org Lett 18: 32703273.

YANG J, LIU XW, WANG DD, TIAN MY, HAN SN, FENG TT, LIU XL, MEI RQ AND ZHOU Y. 2016. Diversity-oriented one-pot multicomponent synthesis of spirooxindole derivatives and their biological evaluation for anticancer activities. Tetrahedron 72: 8523-8536.

YU B, YU DQ AND LIU HM. 2015. Spirooxindoles: Promising scaffolds for anticancer agents. Eur J Med Chem 97: 673-698.

ZHANG B, WANG X, CHENG C, SUN D AND LI C. 2017. Total synthesis of $( \pm)$-corymine. Angew Chem Int Ed 56: 7484-7487.

ZHOU F, LIU YL AND ZHOU J. 2010. Catalytic asymmetric synthesis of oxindoles bearing a tetrasubstituted stereocenter at the C-3 position. Adv Synth Catal 352: 1381-1407.

ZHOU F, TAN C, TANG J, ZHANG YY, GAO WM, WU HH, YU YH AND ZHOU J. 2013. Asymmetric copper(I)catalyzed azide-alkyne cycloaddition to quaternary oxindoles. J Am Chem Soc 135: 10994-10997.

ZIARANI GM, GHOLAMZADEH P, LASHGARI N AND HAJIABBASI P. 2013. Oxindole as starting material in organic synthesis. Arkivoc (i): 470-535. 